Draft version December 17, 2018

Typeset using LATEX twocolumn style in AASTeX61

\title{
THE DISCRETENESS-DRIVEN RELAXATION OF COLLISIONLESS GRAVITATING SYSTEMS: ENTROPY EVOLUTION IN EXTERNAL POTENTIALS, N-DEPENDENCE AND THE ROLE OF CHAOS
}

\author{
Leandro Beraldo e Silva, ${ }^{1,2}$ Walter de Siqueira Pedra, ${ }^{3}$ Monica Valluri, ${ }^{2}$ Laerte Sodré, ${ }^{1}$ And \\ JEAN-BERNARD BRU ${ }^{4,5,6}$
}

\footnotetext{
${ }^{1}$ Universidade de São Paulo, Instituto de Astronomia, Geofísica e Ciências Atmosféricas, Departamento de Astronomia, CEP 05508-090, São Paulo, SP, Brasil

${ }^{2}$ Department of Astronomy, University of Michigan, Ann Arbor, MI 48109, USA

${ }^{3}$ Universidade de São Paulo, Instituto de Física, Departamento de Física Matemática, CP 66318, CEP 05314-970, São Paulo, SP, Brasil

${ }^{4}$ Departamento de Matemáticas, Facultad de Ciencia y Tecnología, Universidad del País Vasco, Apartado 644, 48080 Bilbao, Spain

${ }^{5}$ BCAM - Basque Center for Applied Mathematics, Mazarredo, 14, 48009 Bilbao, Spain

${ }^{6}$ IKERBASQUE, Basque Foundation for Science, 48011 Bilbao, Spain
}

\section{Submitted to ApJ}

\begin{abstract}
We investigate the old problem of the fast relaxation of collisionless $N$-body systems which are collapsing or perturbed, emphasizing the importance of (non-collisional) discreteness effects. We integrate orbit ensembles in fixed potentials, estimating the entropy to analyze the time evolution of the distribution function. These estimates capture the correct physical behavior expected from the 2nd Law of Thermodynamics, without any spurious entropy production. For self-consistent (i.e. stationary) samples, the entropy is conserved, while for non-self-consistent samples, it increases within a few dynamical times, stabilizing at a maximum (even in integrable potentials). Our results make transparent that the main ingredient for this fast collisionless relaxation is the discreteness (finite $N$ ) of gravitational systems in any potential. Additionally, in non-integrable potentials, the presence of chaotic orbits accelerates the entropy production. Contrary to the traditional violent relaxation scenario, our results indicate that a time-dependent potential is not necessary for this relaxation. For the first time, in connection with the Nyquist-Shannon theorem we derive the typical timescale $T / \tau_{c r} \approx 0.1 N^{1 / 6}$ for this discreteness-driven relaxation, with slightly weaker $N$-dependencies for non-integrable potentials with substantial fractions of chaotic orbits. This timescale is much smaller than the collisional relaxation time even for small- $N$ systems such as open clusters and represents an upper limit for the relaxation time of real $N$-body collisionless systems. Additionally, our results reinforce the conclusion of Beraldo e Silva et al. (2017) that the Vlasov equation does not provide an adequate kinetic description of the fast relaxation of collapsing collisionless $N$-body systems.
\end{abstract}

Keywords: galaxies: clusters: general — galaxies: formation — galaxies: halos — galaxies: kinematics and dynamics 


\section{INTRODUCTION}

The relaxation of self-gravitating systems such as globular clusters, galaxies and dark matter halos is characterized by at least two time scales: the crossing (dynamical) timescale $\tau_{c r} \approx R /\langle v\rangle$ and the typically much longer collisional relaxation timescale $\tau_{\text {col }} \approx(N / \ln N) \tau_{c r}$, where $R$ is the system's size, $\langle v\rangle$ is a characteristic velocity and $N$ is the number of bodies - see Binney \& Tremaine (2008).

For small timescales in comparison to $\tau_{\text {col }}$, the collisional relaxation can be neglected, and the system is said to be collisionless. Despite being collisionless, these systems still undergo an initial phase of fast relaxation (in a few dynamical time scales), coined violent relaxation in the scenario proposed by Lynden-Bell (1967). In this scenario, the key ingredient is a time-varying collective potential, which changes individual energies. These indirect energy exchanges among the stars (through the time-varying potential) are further interpreted as driving the relaxation.

Most theoreticians discuss this fast collisionless relaxation in terms of infinitely divisible distribution functions, i.e. in the limit $N \rightarrow \infty$, despite the fact that real stellar systems are composed of a finite $N$ and are not infinitely divisible and smooth. In this context, the kinetic evolution of collisionless systems is traditionally expected to be described by the Vlasov-Poisson equation (with discreteness effects only producing corrections in the long-term evolution through collisional relaxation):

$$
\frac{d f}{d t} \equiv \frac{\partial f}{\partial t}+\vec{v} \cdot \frac{\partial f}{\partial \vec{r}}-\frac{\partial \phi}{\partial \vec{r}} \cdot \frac{\partial f}{\partial \vec{v}}=0
$$

where $f(\vec{r}, \vec{v}, t)$ is the distribution function, representing the probability of finding a test particle in position $\vec{r}$ and velocity $\vec{v}$. In Eq. (1), $\phi(\vec{r}, t)$ is the collective gravitational potential, considered as an external potential for the test particle and self-consistently related to the distribution function by means of the Poisson equation

$$
\nabla^{2} \phi=4 \pi G \int d^{3} \vec{v} f(\vec{r}, \vec{v}, t) .
$$

Eq. (1) yields an evolution for the distribution function $f$ which is time reversible and conserves the entropy $S$. In fact, as shown by Tremaine et al. (1986), defining

$$
S(t) \equiv-\int f \ln f d^{3} \vec{r} d^{3} \vec{v},
$$

then if $d f / d t=0 \Rightarrow d S / d t=0$.

Beraldo e Silva et al. (2017) (hereafter Paper I) ran N-body simulations and studied the evolution of the entropy defined by Eq. (3). This quantity was estimated at each snapshot with well established mathematical prescriptions, and this estimate was shown to have a fast increase in the early stages and a slow, $N$-dependent and almost linear increase in the long-term evolution. This long-term behavior is well described by the orbitaveraged Fokker-Planck equation modelling the collisional relaxation. On the other hand, in Paper I it is argued that, since the Vlasov-Poisson equation implies entropy conservation, the observed entropy increase on a dynamical timescale is an indication of non-validity of the Vlasov-Poisson equation in the violent relaxation.

While there is still no mathematically rigorous proof of the Vlasov-Poisson equation in the full self-gravitating N-body problem, the Vlasov equation has already been proven for any fixed finite time interval and (initially) independently distributed particles subject to an external potential with integrable spatial gradient and bounded interparticle forces, in the limit $N \rightarrow \infty$-see Dobrushin (1979). See also Paper I for a summary of recent mathematical results. Notice that the term "Vlasov-Poisson equation" is used for the special case of a Coulomb twobody potential in self-gravitating systems (Eq. (2) being the corresponding self-consistency condition), whereas "Vlasov equation" refers to generic potentials.

In the current work, instead of focusing on the evolution of a self-gravitating $N$-body system, we study the simpler problem of ensembles of orbits interacting only with fixed external potentials, chosen on the basis of their phase-space properties. As we show below, the entropy evolution in these potentials agrees with what is expected from the 2nd law of Thermodynamics, i.e. it increases whenever the initial state does not correspond to an invariant state compatible with the macroscopic constraints. On the other hand, the entropy evolution of non-self-interacting particles in external potentials raises questions regarding the non validity of the Vlasov equation in a situation where it was expected to be valid. In this work we show that, even though the Vlasov-Poisson equation might be valid in the limit $N \rightarrow \infty$, its use in the description of the evolution of gravitational systems can be problematic for three, correlated, reasons: (1) on physically relevant timescales, the convergence of the discrete, real problem to the continuous limit $N \rightarrow \infty$ can be too slow (i.e. with too weak $N$-dependence), invalidating the use of this continuous limit for timescales of interest and values of $N$ that are typical of real systems; (2) previous rigorous results discussing the validity of the Vlasov equations in the large $N$ limit are not uniform with respect to time (typically, the timescale for which the Vlasov equation is proven to be valid grows only as $\ln N$ - see Boers \& Pickl 2016; Lazarovici \& Pickl 2017), suggesting that in general the Vlasov equation fails to describe the macroscopic state of finite $N$ systems (initially in non-stationary states) 
for sufficiently large times; (3) its physical content is equivalent to the Newtonian description (i.e. at the microscopic level of single trajectories), lacking the physical content necessary to describe emergent phenomena, i.e. on a macroscopic level, particularly the time irreversibility, as expected from a kinetic equation - see $\S 2$ and $\S 9$.

The orbit integration in fixed external potentials carried out in this work allows us to critically revise some of the conclusions drawn in Paper I and to explore in more detail the meaning of the entropy estimators and their relation to the Vlasov equation. This analysis also sheds some light on the fast (violent) collisionless relaxation of $N$-body systems and its time irreversibility. Further, by comparing results obtained for ensembles of orbits in integrable and non-integrable potentials we investigate the role of chaos on the entropy evolution.

The current results supplement those obtained in Paper I, highlighting the origin of the observed rapid entropy increase. Additionally, we confirm the absence of any artificial, non-physical, inputs in Paper I, which might have been introduced by the numerical methods used. In particular, we answer the following questions:

1. Why is there time irreversibility in the fast relaxation of collisionless systems (Paper I) although the equations of motion for individual trajectories are time-reversible?

2. Does the entropy increase observed in Paper I result from truncation errors always involved in the integration of orbits on a computer?

3. Was the observed entropy increase due to artificial correlations introduced by the entropy estimators?

4. Was the observed entropy increase a result of information loss due to coarse-graining when estimating the entropy?

In $\S 2$, we discuss the concept of time irreversibility, making explicit the differences between the descriptions on the micro and macroscopic levels and thereby answer question 1 above. In $\S 3$ we summarize possible ingredients for the time irreversibility of the early collisionless relaxation. In $\S 4$, we introduce the entropy estimator and show its quantitative agreement with the theoretical expression in simple cases. In $\S 5$ we start the study of the dynamical evolution of gravitational systems with the harmonic potential, showing that the numerical scheme is able to recover macroscopic time reversibility when it is present. In $\S 6$ we integrate ensembles of orbits in the Plummer potential, showing that the entropy estimates behave in agreement with the 2 nd law of Thermodynamics, i.e. increasing when the ensemble is far from an invariant distribution and being conserved for an invariant one. These two sections answer questions 2 and 3 above. The role of chaos for time irreversibility is discussed in $\S 7$, where we integrate ensembles of orbits in an ellipsoidal model, studying the $N$-dependence of the entropy evolution. We also perform frequency analysis for these orbits in order to investigate the phase-space structure for the underlying potential models, allowing us to also estimate the fractions of regular and chaotic orbits in each model. Finally, in $\S 8$ and $\S 9$ we discuss the meaning of the entropy estimator and its relation to the Vlasov equation, answering criticism 4 above. We conclude in $\S 10$.

\section{MICRO VS. MACROSCOPIC DESCRIPTION}

Given a system composed of $N$ particles, Classical Mechanics presents a microscopic characterization of its evolution, describing the motion of every single particle in the system, by means of time-reversible equations. Since this generally involves a huge number $(3 N)$ of degrees of freedom, the standard strategy is to reduce this number, by studying quantities characterizing the system as a whole, e.g. total energy, the virial ratio, pressure, temperature, entropy and so on.

The theory par excellence to study such global quantities is Thermodynamics. The 2nd law of Thermodynamics expresses the fact that natural phenomena are generally time irreversible at a macroscopic level, and are parametrized by a quantity which increases with time, the entropy. This agrees with observed phenomena, like diffusion of ink in water or evaporation of a perfume in a room.

Let us emphasize that the distinction between micro and macroscopic levels does not refer to the system size. Instead it refers to the kind of description to be made: description of the movement of each constituent element on the one hand, versus the description of the system as a whole on the other hand. Thermodynamics refers to the macroscopic level and is independent of any specific theory used to model the microscopic behavior. This is illustrated by the fact that it has remained practically intact in the transition from Classical to Quantum and Relativistic Mechanics, and also in face of the new paradigm associated with chaos.

At the same time, when possible, the interpretation of macroscopic phenomena in terms of a mechanical description for individual particles can be illuminating. However, when this is difficult or impossible, it does not mean that the macroscopic effect is not real, but it makes clear the difficulties and limitations of the reductionist point of view: phenomena that occur at 
the macroscopic level generally need to be seen as new phenomena, and not as simple collective manifestations of the microscopic phenomena. In Anderson (1972)'s words: "more is different" (see also Uhlenbeck 1973). From a theoretical point of view, the 2nd law of Thermodynamics can be considered as fundamental as Mechanics, and not as a mere phenomenological consequence of it as discussed e.g. by Bru \& de Siqueira Pedra (2015), in the case of transport theory.

At the end of nineteenth century Boltzmann, although being conscious about the autonomy of the 2nd law of Thermodynamics in respect to Mechanics (see Boltzmann 1974), proposed to link the microscopic properties of a gas, governed by Classical Mechanics, with its thermal behavior expressed by the entropy. In other words, he connected, for the first time, Classical Mechanics with Thermodynamics. This was done through a kinetic equation, which refers to the distribution function $f(\vec{x}, \vec{v}, t)$ in the general format

$$
\frac{d f}{d t}=\Gamma[f],
$$

where the relaxation term $\Gamma[f]$ introduces the irreversibility in the description. The so-called Boltzmann kinetic equation, which applies to rarefied, short-range interacting (collisional) molecular gases, can successfully describe the entropy increase and other transport phenomena for these systems (see Cercignani 1988).

After initially claiming to have derived the 2nd law of Thermodynamics exclusively from Mechanics, Boltzmann had to recognize that his equation contained extra, statistical content. This is not really an artificial feature of his method, but rather a limitation of a purely mechanical description of the evolution of large systems, which cannot, per se, make transparent the irreversible character of the evolution of the macroscopic state expressed by the 2 nd law of Thermodynamics. In other words, an effective description of the macroscopic evolution should take into account, besides Mechanics, statistical ingredients to implement the time irreversibility in the description (see Krylov et al. 1979).

According to Lebowitz (1993), arguments against the physical reality of macroscopic time irreversibility based on the fact that individual particle trajectories are time reversible were already satisfactorily answered in Boltzmann's times. A nice review on this controversy, including references and an answer to the issue, can be found in Lebowitz (2007). In fact, the simple example of a gas expanding in a box is enough to illustrate the point: suppose that all molecules of the gas are initially in a small region around the center of the box with random velocities. Assume also that one could turn off the collisions among the molecules, just allowing for collisions with the walls (let us assume a spherical container). The system would start evolving with each molecule in uniform motion spreading in the box, then colliding with the walls and occupying all the volume. Unless the system is prepared with very specific initial conditions, it will never (or only after an extremely long time) go back spontaneously to the initial state, even if all trajectories are regular. There is macroscopic irreversibility and entropy increase. However, there is no microscopic physical mechanism randomizing the trajectories and driving the system towards a unique, well-defined, equilibrium state. Instead, the system achieves an invariant nonequilibrium state, which can keep some "memory" of the initial state. This is a simple illustration of what is traditionally called phase mixing.

Now, consider a more realistic gas, in which the molecules are allowed to collide amongst each other (in normal conditions, a typical molecule can collide $\approx 10^{6}$ times per second!). The system starts evolving with the molecules elastically colliding with each other and with the walls. After a short time the entire box is filled and the thermodynamical equilibrium is achieved, with the macroscopic state being characterized by the Maxwell-Boltzmann distribution. The system never (or extremely rarely) returns to the initial state with all molecules in the center with the same initial velocities. In this case also there is macroscopic irreversibility and entropy increase. However, now the random collisions drive the system towards a unique equilibrium state independent of the initial condition. This is an extreme example of what is called chaotic mixing, which can occur even for collisionless evolution of ensembles in nonintegrable potentials (see Merritt \& Valluri 1996).

Since the presence of chaos implies the existence of a predictability horizon, i.e. of a time limit beyond which a trajectory cannot be predicted with certainty, chaotic motion can be seen as introducing irreversibility at a $m i$ croscopic level, because reversing the velocities at any instant beyond the predictability horizon, is not guaranteed to recover the initial state. The collisions amongst the molecules in the example above represent an efficient (but non-unique) mechanism to produce chaos in many-body systems.

In the last few decades, significant progress has been made in the study of non-integrable systems (see Lichtenberg \& Lieberman 1992) and some authors support the idea that chaos plays a fundamental role in the macroscopic irreversible evolution. The most radical line of thought in this direction is that of Prigogine (see e.g. Prigogine 1999), who proposes that, in order to explain the observed irreversibility at the macroscopic level, the time irreversibility must be formally present on the mi- 
croscopic level, associated to a fundamental indeterminacy due to chaos, requiring a deep revision of the fundamentals of Classical Mechanics.

On the other hand, according to Lebowitz (1993), even though "instabilities induced by 'locally' chaotic behavior do play a role in determining the nature of the macroscopic evolution (...), the central role in time asymmetric behavior is played by the very large number of degrees of freedom involved in the evolution of macroscopic systems" (see also Lebowitz 1999).

\section{COLLISIONLESS RELAXATION AND CHAOTIC VS REGULAR PHASE MIXING}

Going back to the evolution of collisionless selfgravitating systems, let us remember that their early and fast relaxation is macroscopically time irreversible. In order to avoid confusion about this point, we emphasize that, even if we only consider the $N$-body gravitational problem of dark matter halos in simulations, neglecting any dissipative baryonic component, the process of galaxy formation is macroscopically time irreversible because we only observe this process occurring in one time direction. Besides that, the gravitational $N$ body problem is intrinsically unstable (see Miller 1964; Hemsendorf \& Merritt 2002), and the fast relaxation of collapsing structures is expected to be accompanied by a large amount of chaotic orbits (see Kandrup et al. 2003). Interestingly, Valluri et al. (2007) have found that the presence of chaotic orbits in simulated galaxy mergers seem to be associated mostly to the Miller's instability than to a time-varying potential.

Note also that the violent relaxation scenario proposed by Lynden-Bell (1967) is strongly based on the presence of a time-varying potential. However, the need of such time dependence is criticized by several authors (see Kandrup et al. 1993; Kandrup 1998; Merritt 2005). Additionally, Peñarrubia (2013) argues that it is possible to use a suitable coordinate frame in which the potential remains "static", erasing any dynamical effect of this time dependence.

Moreover, it is well-known that the final state of $\mathrm{N}$ body simulations depends on the initial state, only forming structures resembling the observed ones when starting with cold (low velocity dispersion) initial conditions (see van Albada 1982; May \& van Albada 1984; McGlynn 1984). On the other hand, some seemingly universal properties do emerge from $N$-body simulations, such as the NFW density profile (see Navarro et al. 1997; Navarro et al. 2004) also observed in real systems (see Umetsu et al. 2011) or the pseudo-phase-space density power-law (see Ludlow et al. 2011).
Following this discussion, it is possible to identify at least four different ingredients that can contribute to the macroscopic time irreversibility in the fast collisionless relaxation of $N$-body gravitating systems (although not all of them need to operate simultaneously):

1. a large number of degrees of freedom,

2. phase mixing of particles with a spread of initial conditions,

3. the presence of chaotic mixing,

4. a time-dependent self-consistent potential.

The orbit integration of ensembles in fixed external potentials performed in this work allows us to investigate the possible roles of ingredients 1-3. Interestingly, the results shown in $\S 6$ and $\S 7$, where we clearly observe macroscopic irreversibility even in static and integrable potentials, seem to be in line with the ideas of Lebowitz (1993) quoted above. The important differences introduced by the presence of chaotic orbits are discussed in $\S 7$.

\section{ENTROPY ESTIMATORS}

In Paper I, $N$-body simulations of self-gravitating systems were run, starting with initial configurations far from equilibrium and the evolution of the entropy defined by Eq. (3) was studied. Following well-established mathematical prescriptions, this entropy is estimated as

$$
\hat{S}(t)=-\frac{1}{N} \sum_{i=1}^{N} \ln \hat{f}_{i},
$$

where the integral over the phase-space is translated into a sum over all the particles of the system. Of course, we still have the problem of calculating $\hat{f}_{i}$, the estimate of the distribution function $f$ at the position of each particle $i$. Eq. (5) has been shown to converge to Eq. (3) for $N \rightarrow \infty$ when we calculate $\hat{f}_{i}$ with at least two methods (see Joe 1989; Beirlant et al. 1997; Biau \& Devroye 2015, for rigorous results): the nearest neighbor and the kernel method. In Paper I it is shown that both methods provide very similar entropy evolutions, also similar to that obtained with the EnBiD method (based on a phase-space tessellation into mutually disjoint hypercubes) developed by Sharma \& Steinmetz (2006).

In the nearest neighbor method, $\hat{f}_{i}$ is estimated as the number of particles (one) inside a hyper-sphere of radius $D_{i n}$ around the particle $i$, divided by its volume. Including the normalization factors (see Leonenko et al. 2008), we have generically in $d$ dimensions:

$$
\hat{f}_{i}=\frac{1}{(N-1) e^{\gamma} V_{d} D_{i n}^{d}}
$$


where $\gamma \approx 0.57722$ is the Euler-Mascheroni constant, $V_{d}=\pi^{d / 2} / \Gamma(d / 2+1)$ is the volume of a hyper-sphere of unitary radius and

$$
D_{i n}=\sqrt{\left(\vec{r}_{i}-\vec{r}_{n}\right)^{2}+\left(\vec{v}_{i}-\vec{v}_{n}\right)^{2}}
$$

is the distance in phase-space of particle $i$ to its nearest neighbor $n$. Thus, in 6 dimensions we have

$$
\hat{S}=\frac{1}{N} \sum_{i=1}^{N} \ln D_{i n}^{6}+\ln \left[\frac{\pi^{3}}{6}(N-1)\right]+\gamma .
$$

In Eq.(7), it is assumed that positions and velocities are represented with coordinates that are dimensionless and have similar variances in different directions - see Paper I for more details. In this work, each coordinate is normalized by its initial inter-percentile range containing $68 \%$ of the data around the median.

By means of a tree algorithm, it is possible to optimize the "naive" search for the nearest neighbor, which originally has complexity $N^{2}$, decreasing the complexity to $N \ln N$ - see Friedman et al. (1977). In this work we restrict ourselves to this method due to its speed and illustrative simplicity. For the identification of the neighbors we use the kd-tree algorithm Approximate Nearest Neighbor (ANN) developed by Arya et al. (1998) ${ }^{1}$. The algorithm allows to optimize the search by approximating the nearest neighbor, but we use it without any approximation, identifying the exact nearest neighbor.

In simple cases, it is possible to obtain analytic expressions for the entropy defined by Eq. (3), and to compare them with what we get with the estimator Eq. (8). For example, for an ensemble uniformly sampling a sphere of radius $r_{\max }$ in positions and $v_{\max }$ in velocities, which is used as initial condition in $\oint 7$, the distribution function is independent of the coordinates and the analytic expression for the entropy is

$$
\begin{aligned}
& S_{0}=-\int f_{0} \ln f_{0} d^{3} \vec{r} d^{3} \vec{v}= \\
&=-\ln f_{0}=\ln \left[\left(\frac{4 \pi}{3}\right)^{2} r_{\max }^{3} v_{\max }^{3}\right] .
\end{aligned}
$$

For this configuration, with $N=10^{6}$ the estimator Eq. (8) provides an error of $\approx 4 \%$ relative to Eq. (9).

Another simple case is for a distribution function depending on energy only. In this case, Eq. (3) reduces to

$$
S=-\int_{\phi(0)}^{0} f(E) g(E) \ln f d E,
$$

\footnotetext{
${ }^{1}$ Available at www.cs.umd.edu/ mount/ANN/. A slightly different version, allowing searches in parallel, was developed by Andreas Girgensohn and kindly provided by David Mount.
}

where $E$ is the energy per unit mass, $\phi(r)$ is the gravitational potential and

$$
g(E)=16 \pi^{2} \int_{0}^{r_{m}(E)} d r r^{2} \sqrt{2(E-\phi(r))}
$$

is the density of states. A simple example for which $f=f(E)$ is the Plummer model, characterized by Eqs.(13)-(15) below. With $N=10^{6}$ particles sampling this model, the error provided by Eq. (8) relative to Eq. $(10)$ is $\approx 0.25 \%$. Appendix $\S \mathrm{A}$ discusses the $N$ dependence of the estimator uncertainties.

\section{HARMONIC POTENTIAL AND MACROSCOPIC REVERSIBILITY}

We first study the dynamical evolution of gravitational systems integrating orbits in the harmonic oscillator potential. This allows us to verify if the entropy increase observed in Paper I (and in the results shown below) can be due to spurious truncation errors that could give rise to macroscopic effects and artificially introduce time irreversibility. The harmonic potential is given by

$$
\phi(r)=\frac{1}{2} \Omega^{2} r^{2},
$$

where $\Omega$ is the angular frequency and $r$ is the distance to the center. In this special potential the angular (azimuthal) period, $T=2 \pi / \Omega$, is the same for all particles, independently of their energies. Thus, even though there is phase mixing within one period, after one period each particle is back to its initial position and velocity. Consequently, in addition to the usual microscopic, we also have macroscopic time reversibility.

We start our numerical simulations with $N=10^{6}$ particles sampling a Plummer model, characterized by Eqs. (13)-(15). The sampling and orbit integration in this potential, as well as the others discussed in the following sections, were performed with the Agama Library (Vasiliev 2019). In this section, we integrate orbits in the potential given by Eq.(12) for 30 orbital periods, setting $G M=1$ and $a=1$ in Eqs. (13)-(15). The entropy is then estimated at each snapshot with Eqs. (6)-(8). We repeated this procedure for 10 different realizations and calculated the average of the entropy at each snapshot.

The result is shown in Fig.1. The uncertainties, estimated as the mean standard deviation over 10 realizations, are $\sigma_{\Delta \hat{S}} \approx 0.001$ (smaller than the data points). The entropy keeps oscillating with a constant maximum amplitude (red horizontal line), without any global increase (the difference in amplitude between the last and first peaks is $\approx 10^{-7}$ ). Zooming-in (upper inset plot) helps to visualize the oscillatory pattern. Note that, due to the spherical symmetry, the system returns to the initial macroscopic state after one radial period (when the 


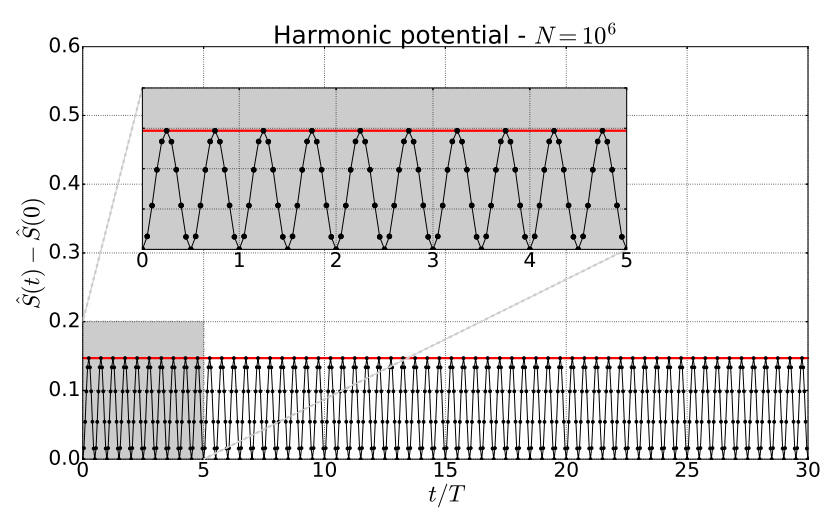

Figure 1. Entropy evolution obtained integrating $N=10^{6}$ orbits in the Harmonic potential, Eq. (12), which, besides microscopic time reversibility, also generates macroscopic reversibility. The horizontal red line shows that there is no net entropy increase and the inset upper plot shows the oscillatory pattern, making clear that the macroscopic time reversibility is perfectly recovered by the numerical scheme.

particles are in positions diametrically opposite to the initial ones), which is half the angular period $T$, and the entropy completes two cycles in each period.

Thus we conclude that for systems whose evolution is macroscopically time reversible (a highly exceptional situation), our numerical procedure is able to perfectly recover this reversibility. This shows that there is no information loss in the orbit integration or in the entropy estimation that could give rise to an artificial entropy production. This simple example also makes explicit the difference between microscopic time reversibility, which is always theoretically present, at the level of single trajectories, and macroscopic time reversibility, which is present in this very particular example but not in general systems such as the ones discussed below.

\section{PLUMMER POTENTIAL AND MACROSCOPIC IRREVERSIBILITY}

We now use the Plummer potential to integrate two different initial conditions. The first is generated with $N=10^{6}$ particles sampling a uniform sphere of radius $a$ and maximum velocity $v_{\max }=\sqrt{2|\phi(a)|}$, where $a$ is the Plummer scale radius of a system of mass $M$. In this case, $v_{\max }=\sqrt{\sqrt{2} G M / a}$ and

$$
\phi(r)=-\frac{G M}{a} \frac{1}{\sqrt{1+(r / a)^{2}}} .
$$

We then integrate these orbits in the potential given by Eq.(13) for 30 crossing times, setting $a=1$ and $G M=1$. The crossing time was estimated as

$$
\tau_{c r}=2 \pi \sqrt{\frac{\left\langle r^{2}\right\rangle}{\left\langle v^{2}\right\rangle}},
$$

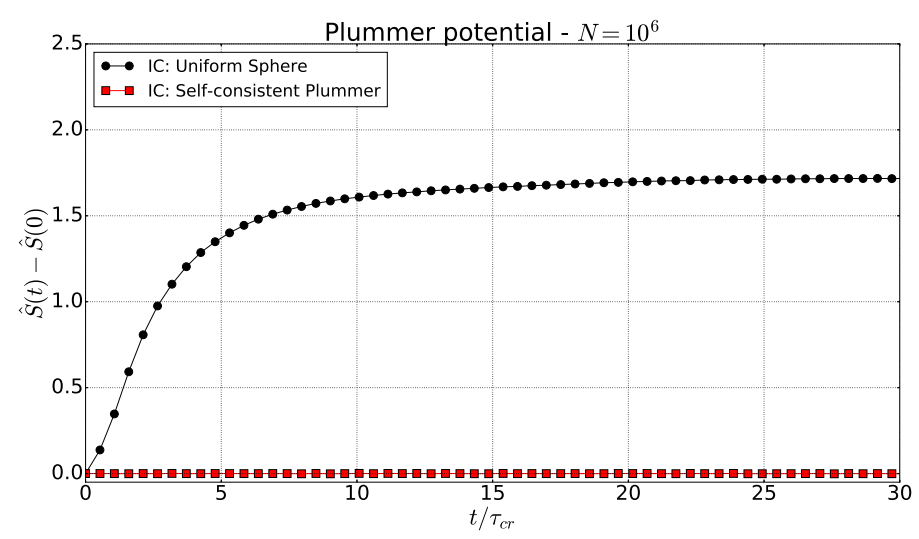

Figure 2. Entropy evolution obtained integrating orbits in the Plummer potential, Eq. (13). Black points: initial condition (IC) sampling a uniform sphere. Red squares: IC sampling a stationary state of the Plummer model, Eq. (15). As expected from the 2nd law of Thermodynamics, when starting far from equilibrium, the entropy increases until it achieves a maximum determined by the constraints. The entropy increases not due to numerical errors since it is correctly conserved when the initial state is stationary.

where the averages are calculated at $t=0$. The entropy is again estimated at each snapshot with Eqs. (6)-(8), repeating the procedure for 10 different realizations and calculating the entropy average at each snapshot. In Appendix A we show the $N$-dependence on both systematic (bias) and statistical errors (normal fluctuations) in the entropy estimators for these and other initial conditions.

The resulting entropy evolution is shown in Fig.2 (black dots). Since the initial condition is far from an invariant state and because the Plummer potential does not share with the Harmonic potential the very particular property of generating macroscopic reversibility, the system evolves through phase mixing and the entropy increases until the system achieves an invariant state, just as expected from the 2nd law of Thermodynamics.

The fact that the entropy increases in a few crossing times is similar to what is observed in Paper I for the evolution of self-gravitating $N$-body simulations. While in the latter case, given the large numbers of particles, two-body relaxation was expected to be negligible for this short timescale, in the present work there is by definition no two-body relaxation, since we integrate independent orbits in an external potential. Note that the entropy increase occurs despite the Plummer potential being time independent and integrable (like any spherically symmetric model), i.e. despite the absence of chaotic orbits. The role of chaos for the time irreversibility is explored in $\S 7$.

The second initial condition was generated by sampling a Plummer model of same mass and scale radius 
as in the Plummer potential used for integration, for which the stationary state is given by (see Aarseth et al. 1974):

$$
f(E)=\frac{24 \sqrt{2}}{7 \pi^{3}} \frac{a^{2}}{(G M)^{5}}(-E)^{7 / 2} .
$$

Since this initial condition represents by definition a stationary state of the potential used for integration, the entropy should be conserved during the evolution of the system. Indeed, Fig.2 shows that the entropy (red squares) is perfectly conserved $\left(\left|\hat{S}\left(30 \tau_{c r}\right)-\hat{S}(0)\right| / \hat{S}(0) \approx 0.015 \%\right)$. This shows again that the entropy increase observed in other configurations is not introduced by information loss due to truncation errors or to errors in the entropy estimation, otherwise an artificial entropy production would be very likely observed even for a stationary state.

\section{ELLIPSOIDAL MODEL AND $N$-DEPENDENCE}

Having shown for integrable systems that the entropy estimates have qualitative agreement with the 2 nd law of Thermodynamics and quantitative agreement for initial states where the distribution function is known, we now investigate the entropy evolution in non-integrable ellipsoidal potentials. The $N$-dependence of these estimates sheds light on the role of chaos for time irreversibility.

The model used is defined by the density profile

$$
\rho(m)=\rho_{0} m^{-\gamma}\left(1+m^{2}\right)^{\frac{\gamma-4}{2}},
$$

where $m^{2}=x^{2} / a^{2}+y^{2} / b^{2}+z^{2} / c^{2}, a, b$ and $c$ being the semi-axis in the respective directions, and $\rho_{0}$ is a normalizing factor. We fix the total mass to be $M=1$ and scale $a=1, b / a=0.8, c / a=0.5$. All models considered here are thus triaxial, with a density profile $\rho(m) \propto m^{-4}$ in the external regions. Moreover, when $\gamma=0$, this reduces to the so-called Perfect Ellipsoid, which is fully integrable (i.e. all orbits are regular) despite being triaxial - see de Zeeuw (1985). On the other hand, the introduction of a cusp with inner slope $\gamma>0$ breaks integrability, giving rise to chaotic orbits. Valluri \& Merritt (1998), in a fundamental frequency analysis of orbits evolved in a triaxial Dehnen (1993) model, concluded that the fraction of chaotic orbits increases with the central slope $\gamma$ and that a transition to global stochasticity occurs for $\gamma \gtrsim 2$. The analysis performed in $§ 7.1$ drives us to similar conclusions for the models used here.

We evolve orbits in this model with $\gamma=0$ (Perfect Ellipsoid), $\gamma=1$ (weak cusp) and $\gamma=2$ (strong cusp). These values cover the inner slopes observed in galaxies, dark matter halos and galaxy clusters. We use two different initial conditions: first, a sphere of radius $a$ and maximum velocity $v_{\max }=\sqrt{2|\phi(0,0, a)|}$ uniformly sampled in positions and velocities; second, a Gaussian distribution with standard deviation in the spatial coordinates $\sigma_{r}=a / 3$ and an independent Gaussian distribution for the velocity components with $\sigma_{v}=v_{\max } / 3$, both truncated at $3 \sigma$, (i.e. at $a$ and $v_{\max }$ for spatial and velocity coordinates respectively).

\subsection{The structure of phase-space}

Before investigating the entropy evolution, we study the phase-space structure associated with the models given by Eq. (16). By means of a frequency analysis (in Cartesian coordinates), we identify the fundamental frequencies, resonances and fractions of regular and chaotic orbits (see Valluri \& Merritt 1998; Merritt 1999; Price-Whelan et al. 2016). This can be seen as the microscopic counterpart of the macroscopic characterization made with the entropy estimates. Note, however, that there is no reason to consider the former as more fundamental than the latter. Instead, these can be seen as complementary approaches.

An orbit evolved in an Hamiltonian $H$ with $N$ degrees of freedom can be described with the time evolution of $2 N$ components $\left(x_{1}(t), \ldots, x_{2 N}(t)\right)=$ $\left(q_{1}(t), \ldots, q_{N}(t), p_{1}(t), \ldots, p_{N}(t)\right)$, where

$$
\dot{q}_{k}=\frac{\partial H}{\partial p_{k}}, \quad \dot{p}_{k}=-\frac{\partial H}{\partial q_{k}} .
$$

Each component of a bounded orbit can be written as

$$
x(t)=\sum_{j=1}^{\infty} A_{j} e^{i \omega_{j} t},
$$

where $A_{j}$ are complex amplitudes. If there are at least $N$ isolating integrals of motion, every orbit is regular and restricted to a surface of dimension $\leq N$. In this case, it is possible to apply a global canonical transformation to define angle-action coordinates $(\vec{\theta}, \vec{J})$ such that the Hamiltonian resembles that of free particles, i.e. it only depends on the momenta $\vec{J}: H=H(\vec{J})$. In this way, Eqs. (17) imply that $\vec{J}$ is constant and $\vec{\theta} \propto \vec{\Omega} t$, with constant fundamental frequencies $\vec{\Omega}=\left(\Omega_{1}, \ldots, \Omega_{N}\right)$. Moreover, the frequencies $\omega_{j}$ in Eq. (18) can be written as a linear combination of the fundamental frequencies, i.e $\omega_{j}=\vec{n} \cdot \vec{\Omega}$, where $\vec{n}$ is a vector with $N$ integer components (we will assume a system with $N=3$ degrees of freedom, the 3 spatial coordinates of each orbit).

Computing the Fourier transform of each of the components $x(t)$ integrated over $\approx 100 \tau_{c r}$, it is possible to identify discrete peaks whose locations, combined with the amplitudes $A_{j}$, can be used to obtain the leading 
frequencies $\vec{\Omega}$ (Binney \& Spergel 1982). A fast and accurate technique to calculate the three leading frequencies in any coordinate system was developed by Laskar (1990), and an implementation that uses integer programming (Valluri \& Merritt 1998) is used in this work.

If the coordinate system used to compute the lead frequencies is close to the angle variables associated with an orbit, then the lead frequencies obtained by the above method are the fundamental frequencies (i.e. time derivatives of the angle variables). A previous work (Valluri \& Merritt 1998) has shown that for box orbits, in a Cartesian system with the $x, y, z$ axes aligned with the long, intermediate and short axes of the triaxial ellipsoid, the coordinates are adequately close to the angle variables, and thus $\Omega_{x}, \Omega_{y}, \Omega_{z}$ are the fundamental frequencies for box orbits. However for short and long axis tube orbits it is necessary to compute orbital frequencies in symplectic polar coordinates with the symmetry axis of the coordinate system aligned with the symmetry axis of the tube ( $z$ axis for short-axis tubes and $x$ axis for long-axis tubes). If the frequencies of tube orbits are computed in Cartesian coordinates, they appear as lines in frequency maps, although they are not resonances (for more on resonances see below). Therefore, we limit our discussion of frequency maps to box orbits.

A frequency map is a plot showing ratios of these frequencies for each orbit - see Figs. 3-4. In general, the fundamental frequencies are independent. However, for some orbits (resonances), the fundamental frequencies can be such that $\vec{n} \cdot \vec{\Omega}=0$. When two such conditions are satisfied, the orbit is closed (periodic). In a frequency map, stable resonances appear as filled straight lines, while unstable resonances appear as void lines.

In an integrable Hamiltonian $H^{0}$, the fundamental frequencies of each orbit are uniquely determined by its initial conditions and are conserved. In this case, in general initial conditions, resonances appear only "by chance", representing a set of measure zero. In a non-integrable model generated by a small perturbation of $H^{0}$, it is common to observe the phenomenon of resonance trapping, in which orbits close to a resonant condition get "captured" by the resonance (Binney \& Tremaine 2008). A consequence of resonance trapping is a further restriction of the phase-space region explored by the orbit. The relation of this effect to relaxation is discussed in $\S 7.3$.

A related important aspect of resonances for the phase-space structure can be illustrated by the KolmogorovArnold-Moser (KAM) theorem (see Lichtenberg \& Lieberman 1992). According to it, if an integrable Hamiltonian is perturbed, the very non-resonant orbits maintain their topological properties, i.e. remain quasiperiodic. The same happens for orbits close to stable resonances. On the other hand, orbits close enough to unstable resonances can be drastically modified, giving rise to stochastic motion even for a small perturbation (thus, resonances can be seen as seeds of stochasticity). With a small perturbation, stochastic regions are separated from each other, and when the perturbation is increased these regions tend to grow and to overlap, eventually transitioning to global stochasticity (see Ford 1975; Chirikov 1979; Lichtenberg \& Lieberman 1992).

While for regular orbits the fundamental frequencies identified by the algorithm are conserved, this is not the case for chaotic orbits. ${ }^{2}$ In order to classify the orbits, this fact can be explored by computing the fundamental frequencies in two consecutive time intervals $T_{1}$ and $T_{2}$. For each orbit we compute

$$
\Delta \nu_{i}=\frac{\Omega_{i}\left(T_{2}\right)-\Omega_{i}\left(T_{1}\right)}{\Omega_{i}\left(T_{1}\right)},
$$

where $i$ refers to each component. Then we define the "frequency drift" $\log (\Delta \nu)$ as the largest among the three. The larger $\log (\Delta \nu)$, the more chaotic the orbit.

We evolve $N=10^{4}$ orbits for $\approx 100 \tau_{c r}$, recording their phase space coordinates at $10^{5}$ equally spaced time steps $^{3}$. Then, the box orbits are selected. Fig. 3 shows the frequency map obtained with the uniform sphere initial conditions in the three models $\gamma=0,1,2$, color coded by the value of $\log \Delta \nu$. As expected, the Perfect Ellipsoid essentially generates only regular orbits, showing that the numeric scheme for orbit integration and frequency identification is accurate. Larger inner slopes $\gamma$ produce increasing fractions of chaotic orbits, in agreement with the conclusions drawn by Valluri \& Merritt (1998) for a triaxial Dehnen model.

The presence of several resonantly trapped orbits is clearly evident as straight lines in the frequency maps of the two non-integrable models $\gamma=1,2$ (recall that resonant orbits satisfy a condition like $l \Omega_{x}+m \Omega_{y}+n \Omega_{z}=0$, where $l, m, n$ are integer numbers). In the weak cusp

\footnotetext{
${ }^{2}$ Although the leading frequencies in Cartesian coordinates are not fundamental frequencies for tube orbits, they are still conserved since the true fundamental frequencies are linear combinations of the lead frequencies. Hence in what follows we focus on fundamental frequencies.

3 Valluri \& Merritt (1998) showed that increasing the integration time and decreasing the time spacing in the time series increases the accuracy for the recovered orbital frequencies in an integrable potential; for our current orbit integrations, $\log (\Delta \nu)<-4$ for the majority of orbits in the Perfect Ellipsoid which should contain only regular orbits. For a fraction of orbits with longer orbital periods than the average $\tau_{c r}$ and very small fundamental frequencies the accuracy with which the numerical scheme recovers the frequencies is lower. This can be the reason for the appearance of hints of resonances in the left panels of Figs. 3 and 4 .
} 
model $(\gamma=1)$ we see numerous stable resonances which appear as clusters of points along straight lines. It is clear that they are stable because their diffusion rates (as indicated by the colors of the points) are small (typically $\log (\Delta \nu) \lesssim-3)$. The orange points scattered along a line with a slope of approximately unity on the righthand side of the maps correspond to chaotic box orbits which are associated with the stochastic layer (separatrix) between the family of short-axis tubes with $\Omega_{x} \sim \Omega_{y}$. Similarly the clusters of orange points at $\Omega_{y} / \Omega_{z} \sim 1$ and $\Omega_{x} / \Omega_{z} \sim 0.75$ arise from the chaotic box orbits associated with the stochastic layer between the inner and outer long-axis tubes, while those at $\Omega_{y} / \Omega_{z} \sim 1$ and $\Omega_{x} / \Omega_{z} \sim 0.9$ are associated with the stochastic layer between the outer long-axis tubes and the short-axis tubes.

Previous studies have shown that when an integrable potential is perturbed by a central density cusp (as in this paper and Valluri \& Merritt 1998), or a central supermassive black hole or figure rotation (Deibel et al. 2011), both the strength and number of resonances increase. As the strength of the perturbation increases (e.g. from $\gamma=1$ to $\gamma=2$ ), the resonances grow stronger and begin to overlap. This is accompanied by an overlap of the separatrices surrounding the resonances (which contain hyperbolically unstable orbits). Resonance overlap is a well known cause of global chaos in Hamiltonian systems (Chirikov 1979) and may be thought of as occurring when several different resonances compete to trap the same orbit (Binney \& Tremaine 2008). This accounts for the fact that the frequency map for the $\gamma=2$ model appears to have very few regular regions and is largely occupied by chaotic orbits.

Fig. 4 shows the frequency map for the box orbits selected from the sample with the Gaussian initial conditions, again color coded by $\log (\Delta \nu)$. The qualitative behavior is very similar to the previous case: in the Perfect Ellipsoid all the orbits can be safely classified as regular, while larger inner slopes $\gamma$ generates increasing fractions of chaotic orbits. As in the previous case, the weak cusp model $\gamma=1$ shows a prominence of resonance lines, which are destroyed in the strong cusp model $\gamma=2$.

In Fig. 5 we show the cumulative distribution function for the frequency drift $\log (\Delta \nu)$ in the three models $(\gamma=0,1,2)$ and for the two initial conditions (different colors). This plot summarizes the main conclusion from the previous results: the introduction of larger inner slopes produces increasing fractions of chaotic orbits. Additionally, Figs. 3-4 show that a large fraction of orbits get trapped in resonances when evolved in the weak cusp model $\gamma=1$. Having established these results, we now study the entropy evolution in these models, integrating the two initial conditions for $300 \tau_{c r}$, with numbers of orbits ranging from $N=10^{4}$ to $N=10^{8}$.

\subsection{Entropy evolution: uniform initial conditions}

The data points in Fig. 6 show the entropy evolution for orbits integrated in the three potential models $(\gamma=0,1,2)$ with the uniform sphere initial condition. Different colors represent ensembles with different numbers of orbits. The points represent an average over 10 realizations for $N \leq 10^{6}$, but only one for $N \geq 10^{7}$.

In all models the entropy increases rapidly, achieving a maximum after $\approx 10-50 \tau_{c r}$. The maximum entropy value is different for each model, which is not surprising since the phase-space volume accessible to each ensemble depends on the model and the nature of the orbits comprising the ensemble. In the integrable potential $(\gamma=0)$, each orbit explores the entire surface of a torus in phase-space. For $\gamma=1,2$ we have significant fractions of chaotic orbits and since such orbits (in a Hamiltonian potential) only conserve one integral of motion (energy) they will undergo rapid chaotic mixing (in $\sim 30-100 \tau_{c r}$ as pointed out by Merritt \& Valluri (1996)) to fill the 5-dimensional phase space surface defined by the energy. In practice in most non-integrable potentials, orbits remain trapped in lower dimensional regions of phasespace defined by resonances (the so called Arnold web) that do not correspond to a unique state associated with thermodynamical equilibrium, or even to a stationary state self-consistently related to the potential.

The data points in Fig. 6 are fitted by the function

$$
\begin{aligned}
\Delta \hat{S}= & \frac{A}{\pi / 2+\arctan (B C)} \times \\
& \times\left\{\arctan \left[B\left(t / \tau_{c r}-C\right)\right]+\arctan (B C)\right\},
\end{aligned}
$$

where parameters $A, B$ and $C$ represent respectively: the final entropy increase, the relaxation rate and the time delay (in units of $\tau_{c r}$ ) for entropy production, i.e. the time at which the entropy starts to increase. The terms $\arctan (B C)$ and $\pi / 2+\arctan (B C)$ ensure that $\Delta \hat{S}(t=0)=0$ and that $A=\Delta \hat{S}(t \rightarrow \infty)$, respectively. Note that $C$ gives an upper bound on the timescale for which the entropy evolution is approximately compatible with the Vlasov equation, which yields no entropy production. This point is discussed in $\S 9$.

Eq. (20) provides a reasonable fit for ensembles of all sizes $N$ in various models, as shown by solid lines in Fig. 6. These fits allow us to study the $N$-dependence of parameters $A, B$ and $C$. Fig. 7 shows that the parameter $A$ is nearly constant, whereas $B(N)$ and $C(N)$ can be fitted by power laws, which are used to predict the behavior for a typical number of stars in a galaxy, $N=10^{11}$ (dashed lines in Fig. 6). Since the nature of 


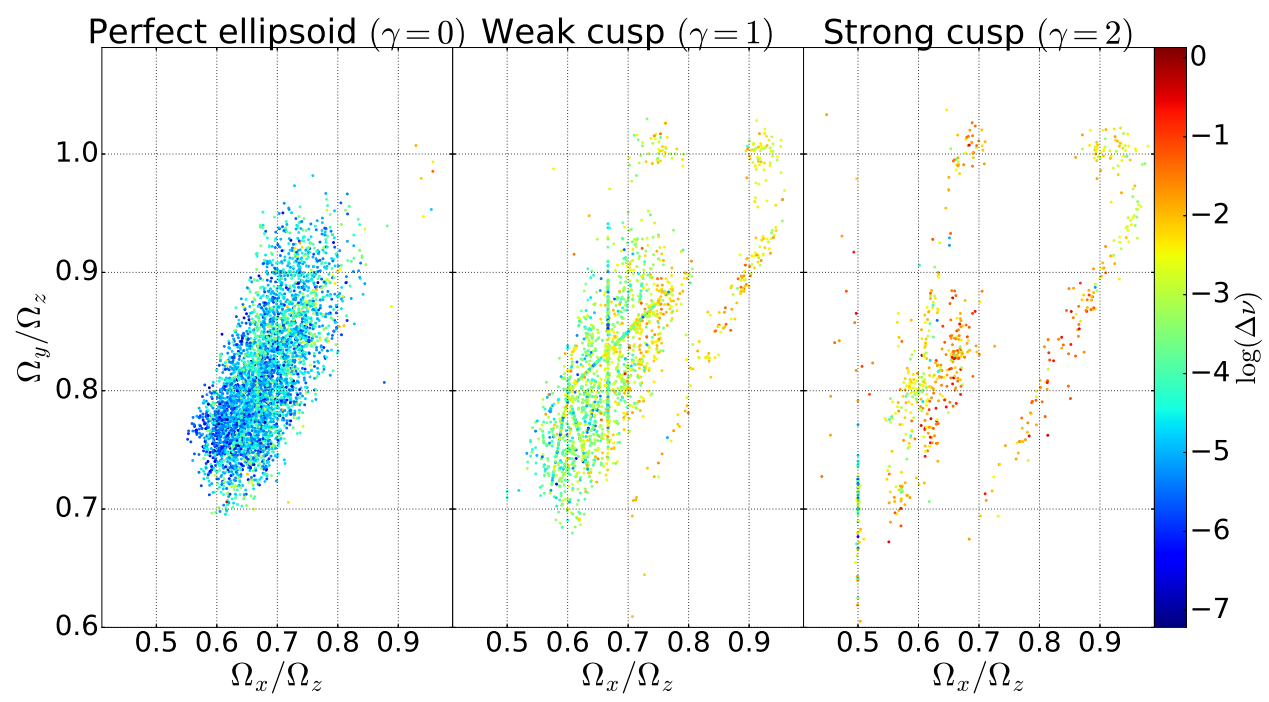

Figure 3. Frequency map of the box orbits selected from $10^{4}$ orbits with the uniform sphere initial conditions, for the three models $\gamma=0,1,2$, color coded by $\log \Delta \nu$. The Perfect Ellipsoid only generates regular orbits (small $\Delta \nu)$, while larger inner slopes $\gamma$ generates increasing fractions of chaotic orbits. Note also the prominence of resonant lines in the weak cusp model.

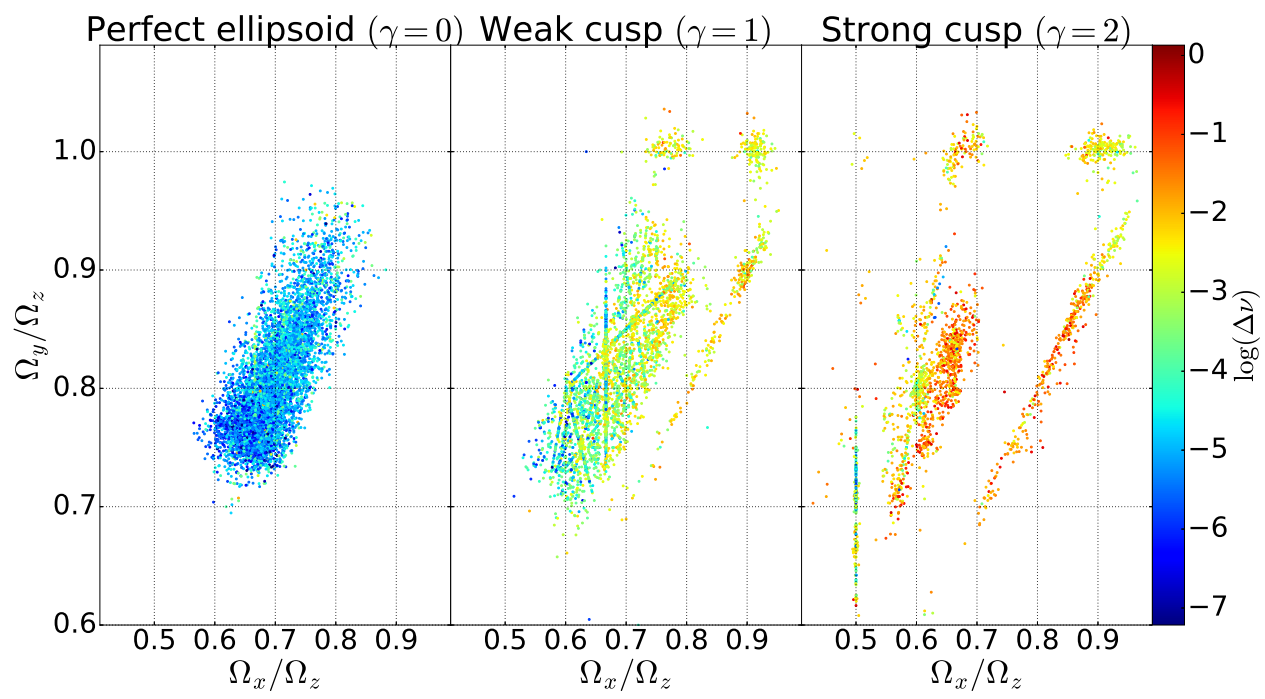

Figure 4. Similar to Fig. 3, but now for the Gaussian initial conditions. Once more, the Perfect Ellipsoid only generates regular orbits, while larger inner slopes $\gamma$ generate increasing fractions of chaotic orbits. Note again the prominence of resonant lines in the weak cusp model.

dark matter, i.e. its constitution, is still completely unknown, with candidates ranging from ultralight bosons to massive primordial black holes (see Bertone \& Tait 2018), we do not speculate here about its number in a typical galaxy.

The parameter $B$ is smaller (and $C$ is larger) for the Perfect Ellipsoid ( $\gamma=0$, integrable) than for the strong cusp model $(\gamma=2$, non-integrable, hosting large fractions of chaotic orbits, as shown in $\S 7.1)$. This indicates that the presence of chaotic orbits anticipates and increases the rate of entropy production.
We estimate the typical relaxation time as the time $T_{\Delta S / 2}$ when the entropy achieves half of its asymptotic value $A$. Substituting this definition in Eq.(20), we have

$$
\frac{T_{\Delta S / 2}}{\tau_{c r}}=\sqrt{B^{-2}+C^{2}} .
$$

The points in Fig. 8 represent this quantity, calculated with the best fit values of parameters $B$ and $C$. These points can be well fitted by power laws

$$
T_{\Delta S / 2} / \tau_{c r} \propto N^{\alpha / d}
$$




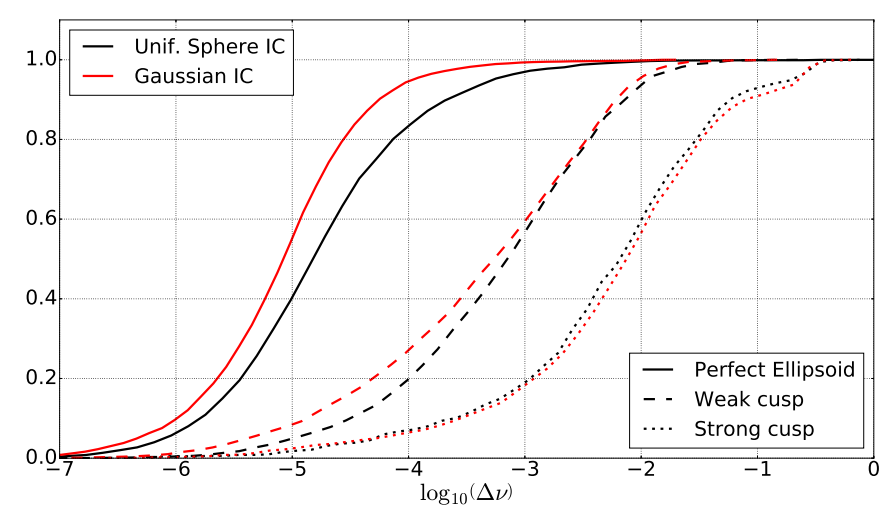

Figure 5. Cumulative distribution function for the frequency drift $\log \Delta \nu$. Larger inner slopes $\gamma$ produces increasing fractions of chaotic orbits.

where $d=6$ is the dimension of the phase-space (the reason for writing Eq. (22) in this format will be clear below). These power law fits are shown as black lines in Fig. 8. For all models the entropy has a significant increase after $1-10 \tau_{c r}$, even in the extrapolation to $N=10^{11}$. Note that smaller values of $T_{\Delta S / 2}$ represent an earlier entropy production, and once more we conclude that the presence of chaotic orbits anticipates it.

In Beraldo e Silva et al. (2018 submitted) a simple connection between the entropy evolution and the NyquistShannon theorem is shown. This theorem states a one-to-one correspondence between a function in a $d$ dimensional continuum and a discrete sample of it if the number of sampling points is $N \gtrsim K^{d}$, where $K$ is the function bandwidth (i.e. the inverse size of its smallest substructures). Conversely, given a sample of size $N$ the theorem states that only functions with

$$
K \lesssim N^{1 / d},
$$

i.e. with structures not too fine, can be uniquely associated to the sample.

Beraldo e Silva et al. (2018 submitted) have shown that the entropy estimated with Eqs. (5)-(6) is in good agreement with the Nyquist-Shannon criterion, Eq. (23). As a consequence, the power law for the relaxation time, Eq.(22), can be seen as a natural consequence of the connection with the Nyquist-Shannon theorem. For a system with phase-space structures evolving in such a way that the bandwidth $K$ of the distribution function grows linearly with time, we would expect $\alpha / d=1 / 6 \approx$ 0.166 . Note the striking value $\alpha / d \approx 0.163 \rightarrow \alpha \approx 0.98$ obtained for the Perfect Ellipsoid. For the weak and strong cusp models, the power laws correspond to $\alpha \approx$ 0.91 and $\alpha \approx 0.85$, respectively (see Fig. 8).

For long-range interacting systems in $d=2$, Pakter \& Levin (2017) also found power laws for the $N$ - dependence of the typical time for entropy increase, with $\alpha=1$ in an integrable model. This result also fits in the association with the Nyquist-Shannon criterion, Eq.(23), for a bandwidth increasing linearly with time. Interestingly, Pakter \& Levin (2017) also found a weaker $N$-dependence (smaller $\alpha$ ) for non-integrable systems, which they interpret as a consequence of the development of more complex phase-space structures in the presence of chaotic motion. The weaker $N$-dependence obtained here for the non-integrable models $\gamma=1,2$ (Fig.8) seems in line with this. As shown by Beraldo e Silva et al. (2018 submitted), this weakening of the $\mathrm{N}$-dependence in non-integrable models is associated to a bandwidth growing (i.e. fine structures developing) faster than linearly in time for these non-integrable models.

\subsection{Entropy evolution: Gaussian initial conditions}

Fig. 9 shows the entropy evolution for ensembles of orbits with the Gaussian initial conditions, again in the different models and for various numbers of particles. The qualitative behavior is similar to that observed for the uniform sphere initial condition, with a fast entropy increase, achieving a maximum after $10-50 \tau_{c r}$.

In contrast with the uniform sphere initial condition, the final entropy amplitude changes non-monotonically from the Perfect Ellipsoid $(\gamma=0)$ to the strong cusp model $(\gamma=2)$. While at first this appears surprising, it is important to remember that the available phase-space volume and the rate at which orbits fill it depend on (a) the geometry of the phase-space and (b) the types of orbits in the ensemble (regular, resonantly trapped or chaotic, and the degree of chaoticity). In addition, the fraction of resonant orbits in a system varies with energy (Deibel et al. 2011). Orbits that are trapped around resonances (either regular resonant or "sticky" chaotic orbits) are confined to a phase space of lower dimensionality than regular or strongly chaotic orbits. As shown in $\S 7.1$, in the weak cusp model $(\gamma=1)$ there is a prominent presence of resonantly trapped orbits. It is interesting to remember that when an orbit is trapped at or near a resonance it is effectively constrained to a phase-space of fewer degrees of freedom and therefore represents a restriction in the phase-space volume explored by the orbit in comparison to the volume it could have explored given its energy. Thus it is natural that a large fraction of resonant orbits will produce a smaller amount of phase mixing and conse- 


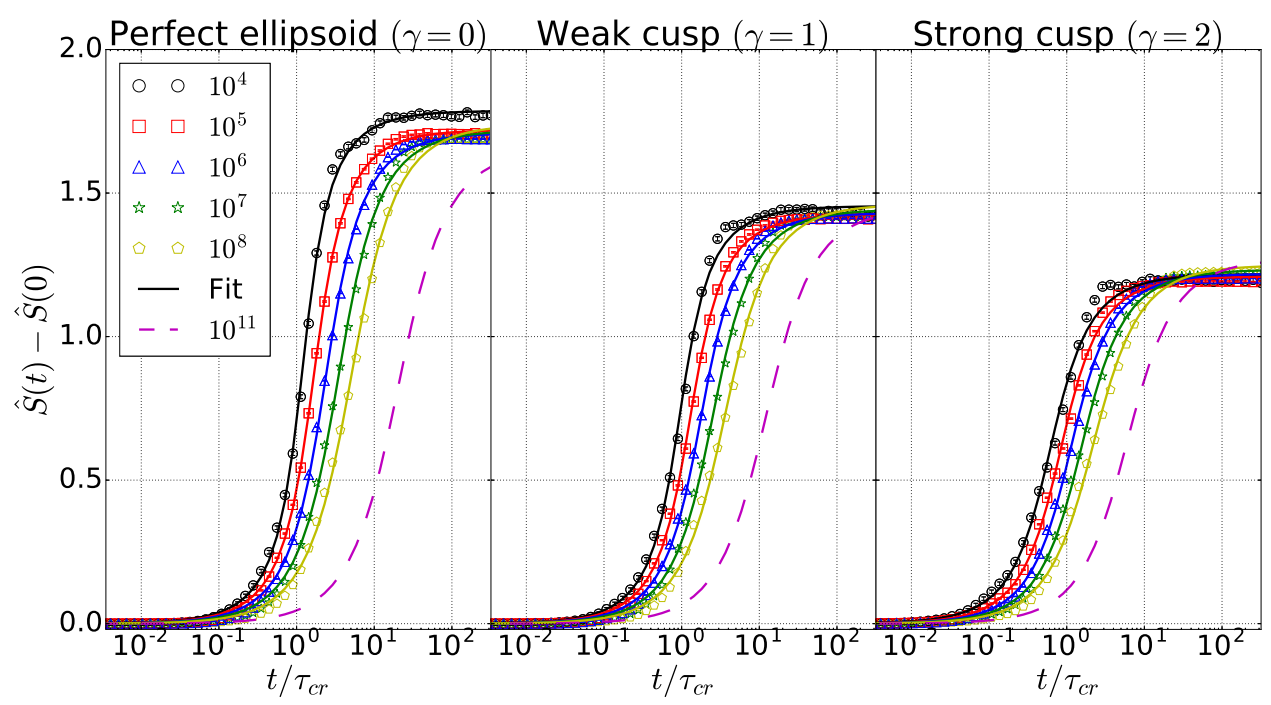

Figure 6. Entropy evolution for the initial uniform sphere evolved in the triaxial model defined by Eq. (16), for inner density slopes $\gamma=0,1,2$. For all models the entropy achieves a maximum in $\approx 10-50 \tau_{c r}$. Solid lines show best fits of Eq. (20), and dashed lines represent the extrapolation to $N=10^{11}$, using the parameters power laws $N$-dependences obtained in Fig. 7 .

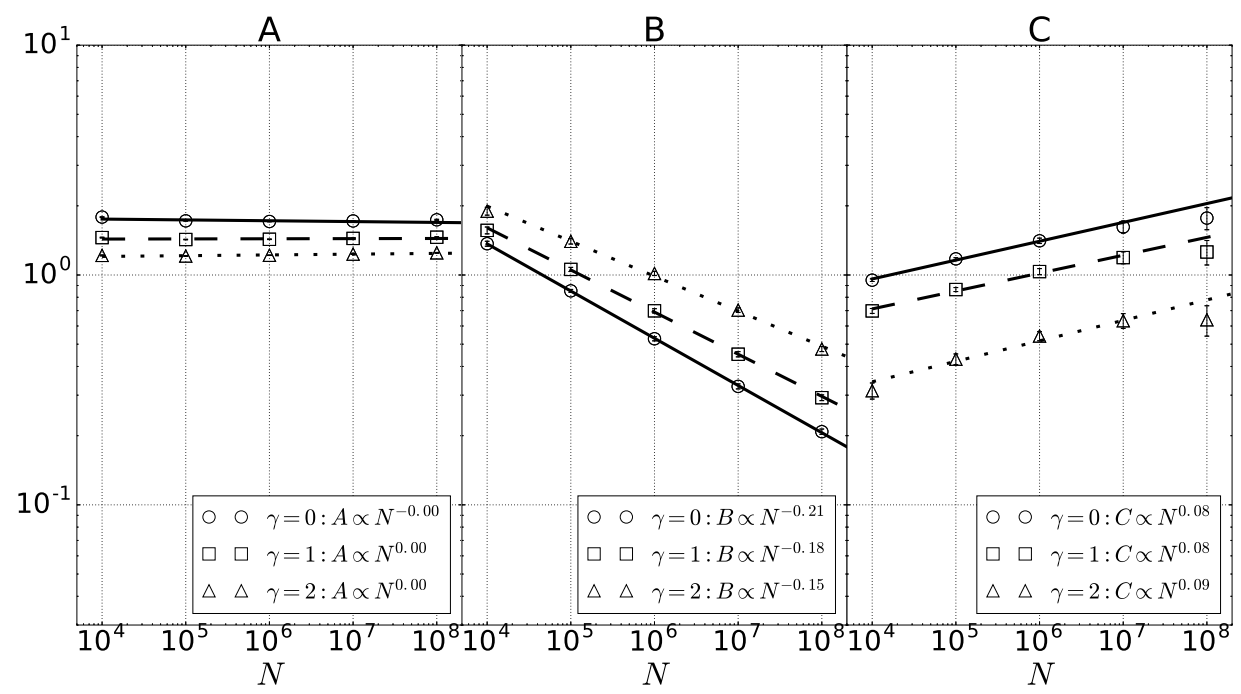

Figure 7. Best fit values of parameters $A, B$ and $C$ in Eq. (20) obtained with data shown in Fig. 6 (initial uniform sphere). Parameters $B$ and $C$ have a power law $N$-dependence. For $B$, the $N$-dependence is weaker in models with larger $\gamma$, i.e. for potentials hosting larger fractions of chaotic orbits.

quently a smaller entropy production, in comparison to non-resonant orbits ${ }^{4}$.

Since the number of resonances increases as one gets deeper in the potential (see Figs. 12 \& 15 of Deibel et al. 2011), and since Gaussian initial conditions result in more orbits exploring the phase-space associated with

\footnotetext{
${ }^{4}$ Interestingly, this suggests that, contrary to the common-sense idea that chaos introduces disorder, a perturbation in an integrable model producing a small amount of chaos can introduce order in respect to the integrable model.
}

a deeper potential, it is not surprising that the entropy production is more severely hampered by resonant trapping. This seems to be the reason for the small entropy production observed for the Gaussian initial conditions integrated in the weak cusp model $\gamma=1$, in comparison to the other models - see Fig. 9. We speculate that this non-monotonicity of the final entropy value as a function of $\gamma$ is not observed for the initial uniform sphere, in Fig. 6, because a smaller fraction of orbits explore the inner region and there is a greater entropy production resulting from phase mixing arising due to 


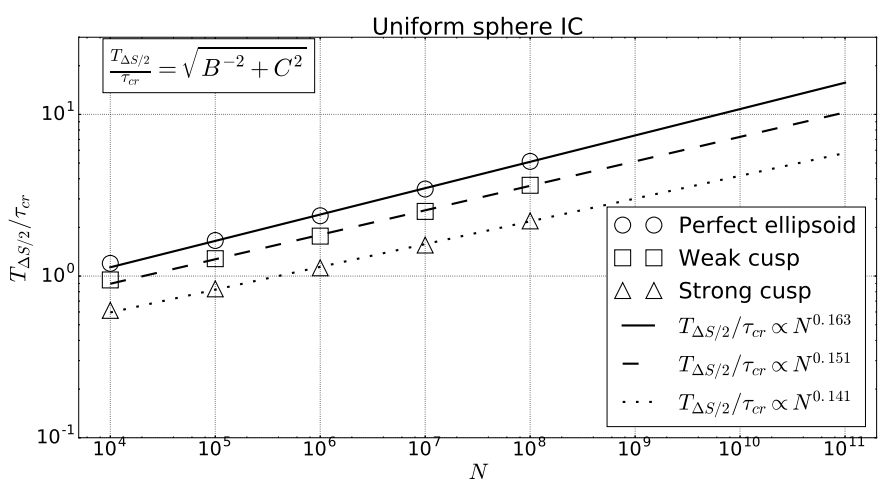

Figure 8. Relaxation time, Eq. (21), for the uniform sphere initial condition. The data are fitted by power laws, $\propto N^{\alpha / d}$, a consequence of the Nyquist-Shannon criterion, Eq. (23). For the Perfect Ellipsoid, $\alpha \approx 0.98$, in agreement for a bandwidth $K$ growing linearly with time for a system in $d=6$ - compare Eqs. (22) and (23). Chaotic orbits produced by the inner cusps accelerate the relaxation and weaken its $N$ dependence.

this much broader spread of initial conditions (note the larger values of the final entropy amplitude in this case).

Again, Eq. (20) provides a reasonable fit to data solid lines in Fig. 9. The $N$-dependencies of parameters $A, B$ and $C$ are shown in Fig. 10. Again parameter $A$ is approximately constant, while parameters $B(N)$ and $C(N)$ can be fitted by power laws, similarly to what we obtained for the uniform sphere initial condition. These power laws are again used to predict the entropy evolution for $N=10^{11}$ (dashed lines in Fig. 9).

With the fitted values of parameters $B$ and $C$, we calculate the relaxation time given by Eq. (21). Fig. 11 shows this quantity for the different models $(\gamma=0,1,2)$, as well as power law fits to these data. These power laws, Eq. (22), can again be seen as related to the NyquistShannon criterion, Eq. (23). In this case the slope implies $\alpha \approx 1.12$, which is reasonably different from the value obtained for the uniform sphere initial condition, expected for a linear time growth of the bandwidth ( $\alpha \approx 1$ ). Besides that, in this case the slope $\alpha$ is the same for the different models $(\gamma=0,1,2)$. The exact reason for this different behavior is not clear, but it seems to be related to the narrowness of the Gaussian initial conditions, in comparison to the much broader uniform sphere. On the other hand, similarly to the uniform sphere, the presence of chaotic orbits seems to accelerate the entropy production (smaller values of $T_{\Delta S / 2} / \tau_{c r}$ ).

Even though there seems to be some differences, the entropy evolution is still similar in the two sets of initial conditions. Specifically, in both initial conditions we conclude that the entropy has a significant entropy increase after $1-10 \tau_{c r}$ for a broad range in $N$ and that this relaxation time scales as $T \propto N^{\alpha / d}$ with $\alpha$ not too different from 1 , even in non-integrable models. It is interesting to contrast this conclusion with the results obtained by Kandrup (1998), where the orbit integration of initially very localized ensembles gives rise to macroscopic evolution occurring in very different rates for phase mixing in comparison to chaotic mixing: the former was observed to evolve with a linear rate and the latter with an exponential rate. However, as pointed out by Merritt (1999), phase mixing of non-localized initial conditions can be much faster, and the similarity of entropy evolution observed in Figs. 6 and 9 seems to be in accordance with this observation.

\section{MEANING OF THE ENTROPY ESTIMATOR}

Since they are applied to finite, discrete samples, the estimators used in this work do not (directly) depend on the distribution function at interparticle phase-space positions, but only on (estimates of) $f$ at the position of each particle, using the information available in its neighborhood. One could argue then that these estimators involve some kind of coarse-graining procedure, meaning that we are averaging the true underlying distribution function in a finite region and thus loosing information that could be completely recovered only in the continuous limit. Note that the same argument is normally used to deny the phenomenon of macroscopic time irreversibility in general. According to this argument, the entropy increase observed here would not represent a real physical effect, but it would be the mere consequence of information loss in the measurement (estimation) process. The time irreversibility is thus relegated to the status of a subjective, non-physical effect.

This solution, however, seems deeply unsatisfactory, since macroscopic irreversibility is an objective fact and cannot depend on our measurement precision (see Jaynes 1965). In particular, a galaxy collapses and forms in one time direction (and not the other) independent of any subjective observational coarse-graining.

In Beraldo e Silva et al. (2018 submitted) it is shown that the entropy estimator used here is in agreement with the Nyquist-Shannon criterion, i.e. that it coincides with the entropy of the assumed distribution function in the continuum, as far as its bandwidth (in frequency/Fourier space) is not too large - see Eq. (23). On the other hand, given a discrete sample, any assumed distribution function with bandwidth larger than this limit value has structures too fine to be realized by the sample and can be seen as an information input. Let us remember that what is given a priori in real systems is a discrete sample, the continuum limit being a mathematical abstraction which can or cannot represent a good 


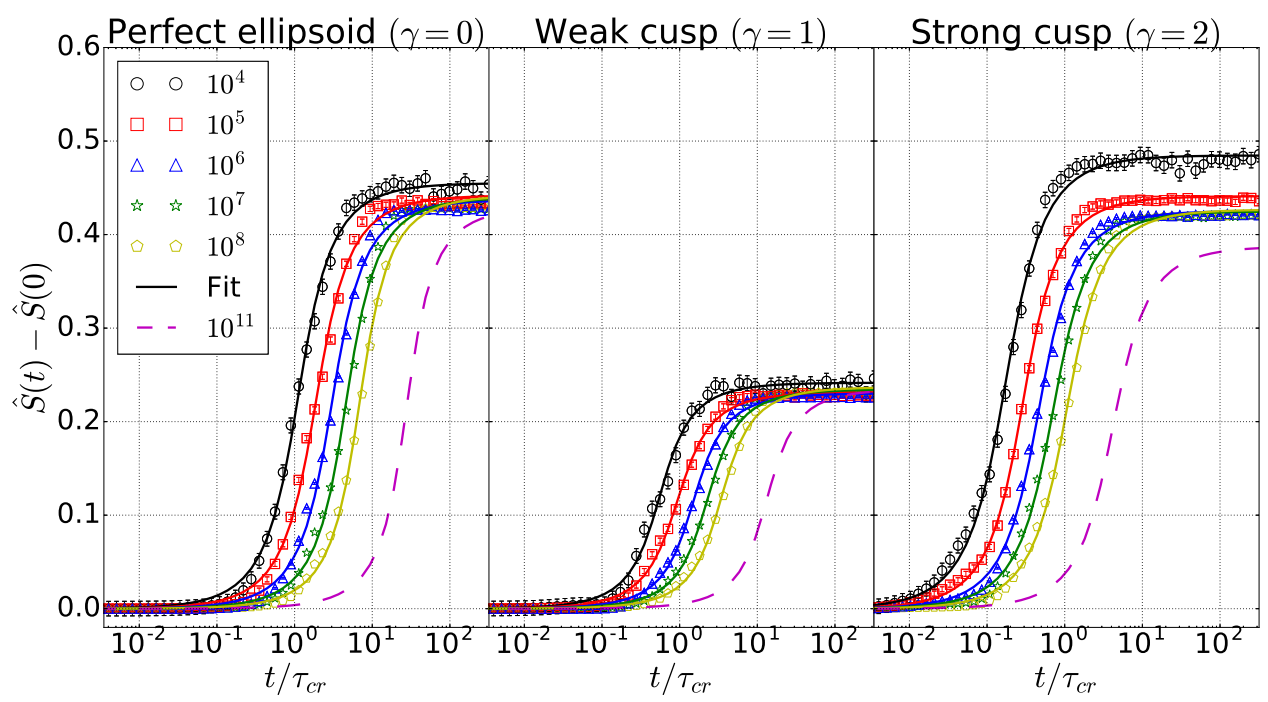

Figure 9. Same as Fig. 6, but using the Gaussian initial conditions. The low entropy production for $\gamma=1$ can be attributed to the large number of orbits trapped by resonances, as shown in Fig. 4.

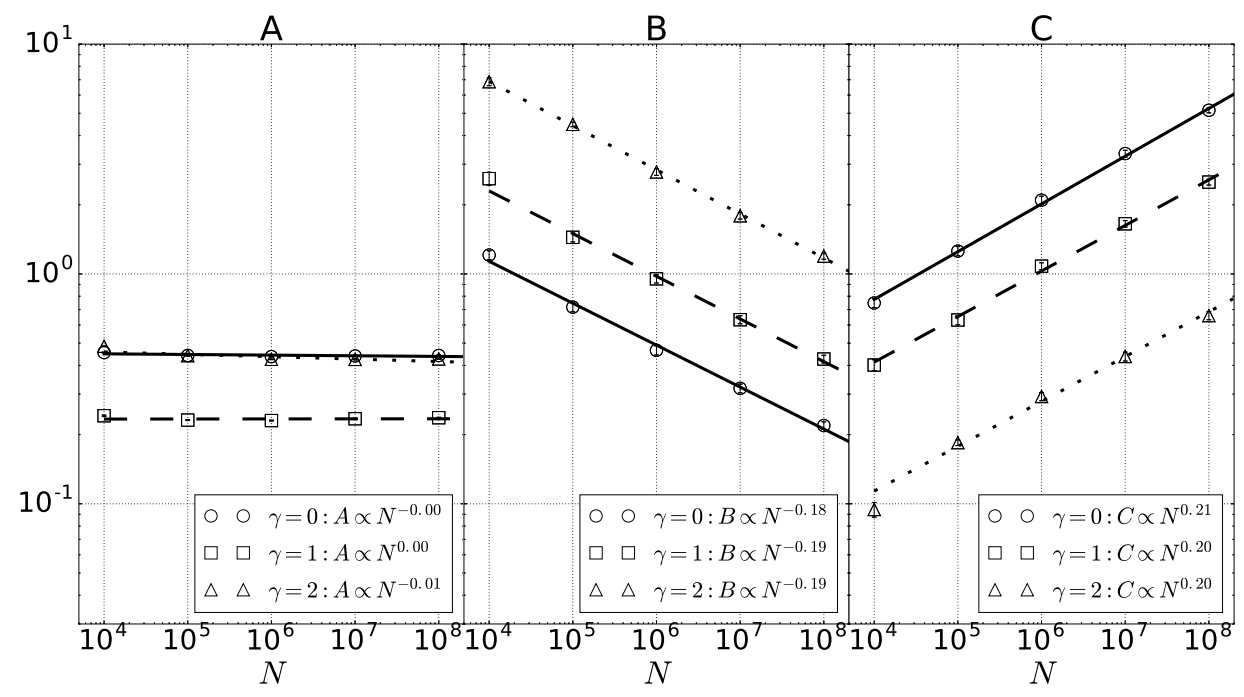

Figure 10. Same as Fig. 7, but using the Gaussian initial conditions.

approximation in the description of the phenomenon. Once this extrapolation to the continuum is done, at a first sight it seems that the entropy estimators produce an information loss. However, these estimators only recover the information we actually have, which is contained in the sample and not in any extrapolation to the continuum.

As noted by Kandrup (1998), gravitational systems are intrinsically inhomogeneous and one important question is whether the number of constituent particles (or stars) suffices for the continuum limit $(N \rightarrow \infty)$ to accurately describe the evolution of the system over the timescales of interest - see also Romero \& Ascasibar (2018). The results of $\S 7$ show that for a finite- $N$ grav- itational system in an external potential the entropy increases significantly after a typical time

$$
T_{\Delta / 2} / \tau_{c r} \approx 0.1 N^{1 / 6}
$$

Thus, even values as large as $N=10^{11}$, representative of the number of stars in real galaxies, are not enough for the continuum limit to be a valid approximation after a few crossing times - see Figs. 6 and 9. This constitutes a real (and fast) relaxation phenomenon and the entropy estimator captures the time irreversibility associated to it. We call it (collisionless) discreteness-driven relaxation, in contrast to the violent relaxation proposed by Lynden-Bell (1967). 


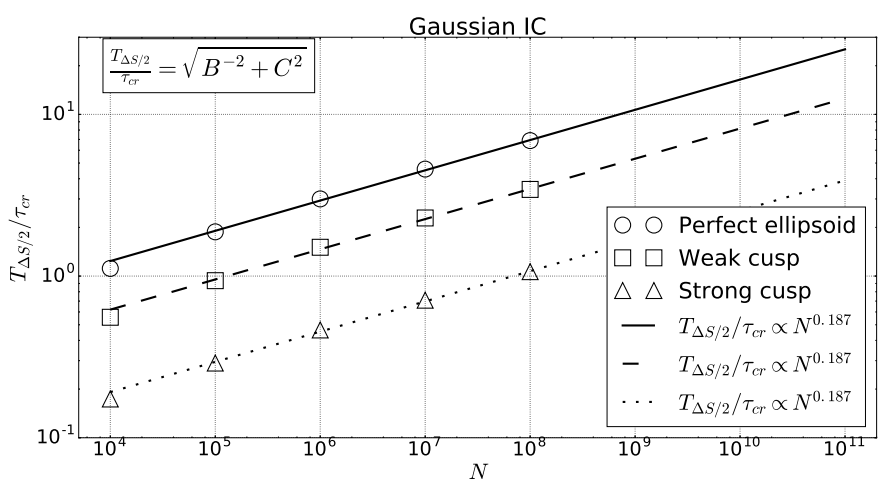

Figure 11. Same as Fig. 8, but using the Gaussian initial conditions. In this case, fitting a power law $\propto N^{\alpha / d}$ we obtain $\alpha \approx 1.12$ for all three models.

\section{COLLISIONLESS RELAXATION AND VLASOV EQUATION}

Having shown that the observed entropy increase is a real effect associated to the intrinsic discreteness of gravitating systems, we move on to the discussion of its kinetic description. This early entropy evolution is similar to what was observed for $N$-body simulations in $\mathrm{Pa}$ per I, in a regime associated to violent relaxation. This phenomenon is traditionally assumed to be described by the Vlasov equation, Eq. (1).

The fundamental problem with this traditional view is that the Vlasov equation is time-reversible and implies entropy conservation (see Tremaine et al. 1986). This already suggests that this equation does not contain the physical ingredients necessary to provide a kinetic description of the fast relaxation of collisionless systems. In fact, Kandrup (1990) has shown that the Vlasov-Poisson equation constitutes a Hamiltonian system with the distribution function being a dynamical variable defined in an infinite-dimensional phase-space. In other words, this suggests that the description provided by the Vlasov equation, at least as it is used in the context of self-gravitating systems, is essentially identical to the microscopic description, i.e. at the level of single trajectories, provided by Classical Mechanics, with possible technical advantages with respect to $N$ body simulations (see Yoshikawa et al. 2013; Colombi et al. 2015; Hahn \& Angulo 2016). However, as a fundamental disadvantage, the Vlasov equation, as any purely mechanical approach, is not able to describe phenomena that only emerge at a macroscopic level, mainly the time irreversibility expressed by the 2nd law of Thermodynamics, which can be considered "one of the most perfect laws in physics" (Lieb \& Yngvason 1999) and has never been faulted by reproducible experiments.
Additionally, all the rigorous mathematical results on the Vlasov equation for a given finite- $N$ system are only able to prove its validity for a finite time - see Boers \& Pickl (2016); Lazarovici \& Pickl (2017). It is thus not surprising that this property is numerically verified, as also reported by Farias et al. (2018) (appeared during the revision process of the current paper), where the polynomial behavior in $N$ for the validity time of the Vlasov equation is also independently emphasized, albeit without any conceptual explanation nor a precise study on the polynomial law (as done by Beraldo e Silva et al. 2018 submitted). At this point, recall that the convergence of the effective distribution function to its thermodynamic limit $N \rightarrow \infty$ is not uniform with respect to time, in all mathematically rigorous results on the validity of the Vlasov equation. That is, even if one can approximate arbitrarily well the effective distribution for a $N$-body system via a solution of the Vlasov equation, for some fixed sufficiently large $N_{0}$ and on a fixed time interval, say $[0, T]$, this function would not anymore, in general, well approximate the effective distribution of the finite system for times $t>2 T$ (the new $N$ necessary to provide a good approximation on $[0,2 T]$ is usually $\gg N_{0}$ ). In other words, everything depends on the involved time- and $N$-scales of the system under consideration. If one studies 11 of liquid, corresponding to $N=10^{24}$ (cf. the Avogadro constant), for times of order of years, the thermodynamic limit $N \rightarrow \infty$ seems to be perfectly justified, at least empirically. This is much more questionable for gravitating systems such as galaxies, with typically $N \lesssim 10^{11}$ and ages of $\approx 10^{10}$ years, unless one dogmatically postulates that such objects are well-described by the limit $N \rightarrow \infty$. In fact, criticizing such a postulate is a central point here, as well as in Paper I and Beraldo e Silva et al. (2018 submitted). What is more, even in the continuous limit $N \rightarrow \infty$, the very notion of large-time convergence of a sequence of distribution functions developing rapidly varying structures (filaments) with the time evolution is a non-trivial conceptual point: such sequences cannot converge in the point-wise sense and the so-called weak convergence is a more natural notion in this situation, as pointed out by Mouhot \& Villani (2011). This type of convergence means, roughly, that structures that get arbitrarily fine in the limit must be "averaged out" in order to obtain a well-defined limiting distribution. It is well-known that the entropy is not continuous with respect to such a weak convergence and therefore, an entropy production for the finite system is clearly not in contradiction with the constant-entropy of the Vlasov equation at any fixed, finite time. Our approach (see in particular Beraldo e Silva et al. 2018 submitted) sheds 
some light on this question, by providing a quantitative criterion to objectively evaluate the "collapse" of fine structures of distributions of particles of macroscopic systems, at fixed (finite) $N$.

Let us stress that although we evolve the orbits in a fixed potential, we do so to eliminate the possibility that a time-dependence (which is invoked in violent relaxation scenario proposed by Lynden-Bell 1967) could be affecting the relaxation of the ensemble. This does not mean that in practice the potential fluctuations do not occur or that they do not have any effect on the evolution of the ensemble. Our goal in using a fixed potential is to demonstrate that this discreteness-driven relaxation occurs whenever the phase-space distribution of an ensemble is not in dynamical equilibrium with the potential. By using a fixed potential and an ensemble whose phase-space distribution is not self-consistent with the potential, we show that this alone will cause this relaxation to occur, on short timescales. The potential fluctuations that accompany the formation of real systems will likely accelerate this process.

Thus, even though the early collisionless relaxation of self-gravitating $N$-body systems must be much more complex than the orbit integration explored in this work, our results shed light on the role of its main ingredients: a finite number of particles and the anticipation/acceleration of entropy production in the presence of chaotic motion. Since this early relaxation (together with a time-varying potential) is expected to host a large amount of chaos (see Kandrup et al. 2003), the entropy increase in real $N$-body systems can be even faster, invalidating even sooner the applicability of the Vlasov equation as a description of the macroscopic evolution. Note that this occurs even in absence of any collisional relaxation, and this is why, as already mentioned in $\mathrm{Pa}-$ per I, we prefer not to call Eq. (1) the "Collisionless Boltzmann equation", as suggested by Hénon (1982).

The timescale in Eq. (24), derived with reference to the Nyquist-Shannon theorem by Beraldo e Silva et al. (2018 submitted), can be seen as a theoretical explanation for the fast character of the collisionless relaxation of $N$-body gravitating systems.

\section{CONCLUSIONS}

In this work, we integrate ensembles of orbits in fixed external gravitational potentials, studying the entropy evolution of the ensemble. While this is a much simpler problem than the $N$-body simulations investigated in Paper I, the current analysis capture the essential ingredients for the time irreversibility of its early collisionless relaxation. The conclusions are summarized below:
- The orbit integration in the Harmonic Potential shows that the entropy estimator is perfectly able to recover the macroscopic time reversibility in exceptional cases where it is present. This indicates that this estimator does not introduce any artificial entropy increase, while making clear the difference between microscopic time reversibility (always present in the equations of motion for each particle) and macroscopic time reversibility, present in this potential but not in general models.

- Integration in the Plummer potential shows that, for a non-self-consistent initial condition, the entropy has a fast increase (due to phase mixing), achieving a maximum after $\sim 10 \tau_{c r}$ for $N=10^{6}$. This macroscopic irreversibility occurs despite the potential being static and spherical (integrable). On the other hand, the estimator correctly captures the entropy conservation associated to a selfconsistent (i.e. stationary) sample of the model. This shows again that this estimator does not introduce any artificial entropy increase, behaving in accordance with the 2nd law of Thermodynamics.

- We also investigate a triaxial model whose density profile is $\rho(r) \propto r^{-4}$ in the external regions and has a free parameter $\gamma$ for the inner slope. For $\gamma=0$, this model reduces to the Perfect Ellipsoid, which is integrable. Larger values of $\gamma$ (inner cusps) generate increasing fractions of chaotic orbits, as shown by means of a frequency analysis. This analysis also shows that a large fraction of orbits are resonantly trapped in the weak cusp model $(\gamma=1)$, producing heavily populated resonance lines, which are destroyed in the strong cusp model $(\gamma=2)$.

- We derive a typical relaxation time that scales as $T / \tau_{c r} \propto N^{1 / 6}$ for an initial ensemble sampling a uniform sphere (in positions and velocities) evolved in an integrable potential. Similar $N$-dependencies are found for a different initial condition and for non-integrable models.

- The presence of chaotic orbits seems to accelerate the entropy production (see Figs. 8 and 11), as found by Pakter \& Levin (2017) for $d=2$.

- This power law $N$-dependence of the typical relaxation timescale can be seen as a natural consequence of the Nyquist-Shannon criterion, as pointed out by Beraldo e Silva et al. (2018 submitted). In this way, the key point for macroscopic time irreversibility is the fact that the system is 
discrete, i.e. composed of a finite number $N$ of elements, regardless of the presence of chaotic motion or a time-dependent collective potential, in line with Lebowitz (1993)'s ideas.

- This connection with the Nyquist-Shannon criterion makes clear the objectivity of the relaxation and entropy increase, without need of the subjective idea of information loss due to coarse-graining.

- The derived timescale, Eq. (24), can be seen as an upper limit for the timescale of the collisionless relaxation of real collapsing $N$-body systems, since the collapse (together with a time-varying potential) is expected to produce a large amount of chaotic orbits (see Kandrup et al. 2003), which would tend to accelerate the entropy production.

- Reinforcing the conclusion drawn in Paper I, our results indicate that the Vlasov equation is not able to provide a kinetic description (i.e. in a macroscopic level) of the early collisionless relaxation of gravitating systems.

Some improvements for future work would be a study of the $N$-dependence of the entropy evolution in selfgravitating $N$-body simulations and the prediction of the final entropy value, which could involve identifying the correct constraints in a maximization procedure (see Hjorth \& Williams 2010; Pontzen \& Governato 2013; Beraldo e Silva et al. 2014, for recent attempts).

\section{ACKNOWLEDGEMENTS}

We thank E. Vasiliev for a careful reading and comments. This work has made use of the computing facilities of the Laboratory of Astroinformatics (IAG/USP, NAT/Unicsul), whose purchase was made possible by the Brazilian agency FAPESP (2009/54006-4) and the INCT-A. LBeS is supported by FAPESP (2014/237514 and 2017-01421-0). WdSP is supported by CNPq (308337/2017-4). MV acknowledges support from HSTAR-13890.001, NSF award AST-1515001, NASA-ATP award NNX15AK79G. LSJ is supported by FAPESP (2017/25620-2) and CNPq. JBB is supported by FAPESP (2017/22340-9), by the Basque Government (IT641-13 and BERC 2018-2022 program), and by the Spanish Ministry of Science, Innovation and Universities: BCAM Severo Ochoa accreditation SEV-20170718, MTM2017-82160-C2-2-P. This paper made use of Agama (Vasiliev 2019), ANN (Arya et al. 1998), GSL, matplotlib (Hunter 2007), numpy (Walt et al. 2011) and scipy (Jones et al. 2001-).

\section{REFERENCES}

Aarseth, S. J., Henon, M., \& Wielen, R. 1974, AAp, 37, 183

Anderson, P. W. 1972, Science, 177, 393

Arya, S., Mount, D. M., Netanyahu, N. S., Silverman, R., \&

Wu, A. Y. 1998, J. ACM, 45, 891

Beirlant, J., Dudewicz, E. J., Györfi, L., \& Van Der

Meulen, E. C. 1997, International Journal of

Mathematical and Statistical Sciences, 6, 17

Beraldo e Silva, L., de Siqueira Pedra, W., Sodré, L.,

Perico, E. L. D., \& Lima, M. 2017, ApJ, 846, 125

Beraldo e Silva, L., de Siqueira Pedra, W., \& Valluri, M. 2018 submitted, ApJ

Beraldo e Silva, L., Lima, M., Sodré, L., \& Perez, J. 2014,

Phys. Rev. D, 90, 123004

Bertone, G., \& Tait, T. M. P. 2018, Nature, 562, 51

Biau, G., \& Devroye, L. 2015, Lectures on the Nearest

Neighbor Method, Springer Series in the Data Sciences

(Springer International Publishing)

Binney, J., \& Spergel, D. 1982, ApJ, 252, 308

Binney, J., \& Tremaine, S. 2008, Galactic Dynamics -

Second Edition (Princeton University Press)

Boers, N., \& Pickl, P. 2016, JSP, 164, 1
Boltzmann, L. 1974, Theoretical Physics and Philosophical Problems: Selected Writings, 1st edn., ed.

B. McGuinness, Vienna Circle Collection 5 (Springer Netherlands)

Bru, J.-B., \& de Siqueira Pedra, W. 2015, Mathematical Models and Methods in Applied Sciences, 25, 2587

Cercignani, C. 1988, The Boltzmann Equation and Its Applications, Applied mathematical sciences No. v. 67 (Springer-Verlag)

Chirikov, B. V. 1979, Physics Reports, 52, 263

Colombi, S., Sousbie, T., Peirani, S., Plum, G., \& Suto, Y. 2015, MNRAS, 450, 3724

de Zeeuw, T. 1985, MNRAS, 216, 273

Dehnen, W. 1993, MNRAS, 265, 250

Deibel, A. T., Valluri, M., \& Merritt, D. 2011, ApJ, 728, 128

Dobrushin, R. L. 1979, FAIA, 13, 115

Farias, C. A. F., Pakter, R., \& Levin, Y. 2018, JPA: Math. and Theor.

Ford, J. 1975, Fundamental problems in statistical mechanics III (North-Holland Pub. Co.)

Friedman, J. H., Bentley, J. L., \& Finkel, R. A. 1977, ACM Trans. Math. Softw., 3, 209 
Hahn, O., \& Angulo, R. E. 2016, MNRAS, 455, 1115

Hemsendorf, M., \& Merritt, D. 2002, ApJ, 580, 606

Hénon, M. 1982, AAP, 114, 211

Hjorth, J., \& Williams, L. R. 2010, ApJ, 722, 851

Hunter, J. D. 2007, Computing In Science \& Engineering, 9, 90

Jaynes, E. T. 1965, Am. J. Phys., 33, 391

Joe, H. 1989, AISM, 41, 683

Jones, E., Oliphant, T., Peterson, P., et al. 2001-, SciPy: Open source scientific tools for Python, ,

Kandrup, H. E. 1990, ApJ, 351, 104

Kandrup, H. E. 1998, Annals NY Academy of Sciences, 848,28

Kandrup, H. E. 1998, MNRAS, 301, 960

Kandrup, H. E., Mahon, M. E., \& Smith, Jr., H. 1993, Astronomy and Astrophysics, 271, 440

Kandrup, H. E., Vass, I. M., \& Sideris, I. V. 2003, MNRAS, 341,927

Krylov, N. S., Migdal, A. B., Sinai, Y. G., \& Zeeman, Y. L. 1979, Works on the Foundations of Statistical Physics by Nikolai Sergeevich Krylov (Princeton University Press)

Laskar, J. 1990, Icarus, 88, 266

Lazarovici, D., \& Pickl, P. 2017, Arch. Rat. Mech. Anal., doi:10.1007/s00205-017-1125-0

Lebowitz, J. L. 1993, Physica A, 194, 1

-. 1999, Physica A Statistical Mechanics and its Applications, 263, 516

Lebowitz, J. L. 2007, Boltzmann's Legacy, 63

Leonenko, N., Pronzato, L., \& Savani, V. 2008, TATRA MT. MATH. PUBL., 39, 265

Lichtenberg, A. J., \& Lieberman, M. A. 1992, Regular and Chaotic Dynamics, 2nd edn., Applied Mathematical Sciences No. 38 (New York, NY: Springer-Verlag)

Lieb, E. H., \& Yngvason, J. 1999, Physics Reports, 310, 1

Ludlow, A. D., Navarro, J. F., White, S. D. M., et al. 2011, MNRAS, 415, 3895
Lynden-Bell, D. 1967, MNRAS, 136, 101

May, A., \& van Albada, T. S. 1984, MNRAS, 209, 15

McGlynn, T. A. 1984, ApJ, 281, 13

Merritt, D. 1999, PASP, 111, 129

-. 2005, Annals NY Acad. of Sciences, 1045, 3

Merritt, D., \& Valluri, M. 1996, ApJ, 471, 82

Miller, R. H. 1964, ApJ, 140, 250

Mouhot, C., \& Villani, C. 2011, Acta Math., 207, 29

Navarro, J. F., Frenk, C. S., \& White, S. D. M. 1997, ApJ, 490,493

Navarro, J. F., Hayashi, E., Power, C., et al. 2004, MNRAS, 349, 1039

Pakter, R., \& Levin, Y. 2017, Journal of Statistical Mechanics: Theory and Experiment, 4, 044001

Peñarrubia, J. 2013, MNRAS, 433, 2576

Pontzen, A., \& Governato, F. 2013, MNRAS, 430, 121

Price-Whelan, A. M., Johnston, K. V., Valluri, M., et al. 2016, MNRAS, 455, 1079

Prigogine, I. 1999, Physica A Statistical Mechanics and its Applications, 263, 528

Romero, M., \& Ascasibar, Y. 2018, MNRAS, sty1728

Sharma, S., \& Steinmetz, M. 2006, MNRAS, 373, 1293

Tremaine, S., Hénon, M., \& Lynden-Bell, D. 1986, MNRAS, 219, 285

Uhlenbeck, G. E. 1973, Problems of Statistical Physics, ed. J. Mehra (Dordrecht: Springer Netherlands), 501-513

Umetsu, K., Broadhurst, T., Zitrin, A., et al. 2011, ApJ, 738,41

Valluri, M., \& Merritt, D. 1998, ApJ, 506, 686

Valluri, M., Vass, I. M., Kazantzidis, S., Kravtsov, A. V., \& Bohn, C. L. 2007, ApJ, 658, 731

van Albada, T. S. 1982, MNRAS, 201, 939

Vasiliev, E. 2019, MNRAS, 482, 1525

Walt, S. v. d., Colbert, S. C., \& Varoquaux, G. 2011, Computing in Science and Engg., 13, 22

Yoshikawa, K., Yoshida, N., \& Umemura, M. 2013, APJ, 762,116 


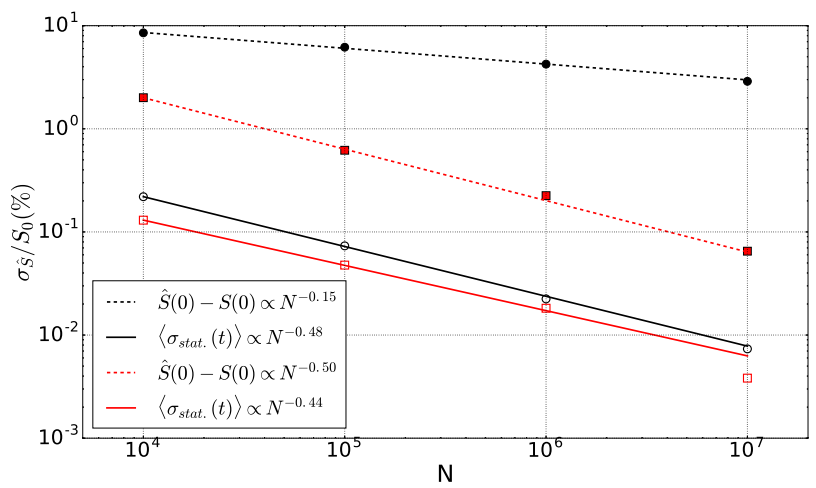

Figure 12. $N$-dependence of errors on the entropy estimates. Black (red) points represent errors obtained with the initial uniform sphere (Plummer sample). Open points are the statistical fluctuation obtained as the mean standard deviation of 10 realizations in each time-step, averaged over all time-steps. These statistical fluctuations behave approximately as $\sim 1 / \sqrt{N}$ (solid lines). Full points represent the error in the initial entropy estimate in comparison with the expected values, Eqs. (9) and (10). The deviation of $1 / \sqrt{N}$ for the uniform sphere is probably due to the non-smoothness in the borders.

\section{APPENDIX}

\section{A. $N$-DEPENDENCE OF ENTROPY ESTIMATOR ERRORS}

In Fig.12 we show the $N$-dependence of the uncertainties $\sigma_{\hat{S}}$ associated with the entropy estimators for these two different initial conditions. As in Fig. 2, black dots are obtained for the initial condition generated from a uniform sphere and the red squares for the initial Plummer sample. Open points represent the statistical fluctuation in the entropy estimate at each time-step obtained with 10 different realizations and then averaged over all time-steps for a fixed $N$. For both initial conditions, we see that these statistical fluctuations approximately behave as $\sim 1 / \sqrt{N}$ (continuous lines) and this is exactly what is rigorously proven, for the case of smooth enough distributions - see Biau \& Devroye (2015).

Full points in Fig. 12 represent the systematic error (bias) in the initial entropy estimate, in comparison to the expected values given by Eqs. (9) and (10). We observe for the initial uniform sphere that these errors are larger and have a decay $\propto 1 / N^{0.15}$ (dashed black line), slower than the rigorously proven $1 / \sqrt{N}$. This is probably due to the fact that the uniform sphere distribution is discontinuous in the border, while, as in the case of the dispersion $\sigma_{\hat{S}}$, the analytic studies of convergence of the estimators assume smoothness in all domain - see Biau \& Devroye (2015). On the other hand, for the Plummer initial sample, this error with respect to the expected initial value behaves as $1 / \sqrt{N}$ (dashed red line). 